\title{
Vortex Pinning and Stability in the Low Field, Superconducting Phases of $\mathrm{UPt}_{3}$
}

\author{
E. Shung and T.F. Rosenbaum \\ The James Franck Institute and Department of Physics, The University of Chicago, Chicago, Illinois 60637 \\ M. Sigrist \\ Yukawa Institute for Theoretical Physics, Kyoto University, Kyoto 606-01, Japan
}

(Received 25 September 1997)

\begin{abstract}
We use an array of miniature Hall probe magnetometers to probe the entry and flow of flux in a single crystal torus of the heavy fermion superconductor $\mathrm{UPt}_{3}$. Local measurements over the hole are exquisitely sensitive to vortex motion anywhere in the torus, and they permit us to detect avalanches restricted to and with a sharp onset in the lower temperature superconducting phase. Computer simulations support a mechanism dependent upon the degenerate nature of the superconducting order parameter. [S0031-9007(97)05197-1]

PACS numbers: 74.70.Tx, 74.25.Dw, 74.60.Ge
\end{abstract}

The lower critical field marks the entry of magnetic flux into a type-II superconductor. With the nucleation of quantized vortices, the Meissner state is partially destroyed and vortices can flow subject to pinning both in the bulk and at the surface. The characteristics of flux penetration and subsequent magnetization relaxation are of particular interest in unconventional superconductors with multiple transitions, where the nature of the barriers and the pinning can depend on the microscopics of the superconducting order.

The most intensively studied example of an exotic superconductor with a double transition is the heavy fermion compound $\mathrm{UPt}_{3}$ [1]. The specific heat in a zero field exhibits two sharp features, one when it enters the superconducting state at $T_{c 1} \sim 0.50 \mathrm{~K}$, followed by a second jump at $T_{c 2} \sim 0.45 \mathrm{~K}$. The lower critical field $H_{c 1}$ has a subtle increase in slope at $T_{c 2}$ [2,3], indicating a gain in the condensation energy due to the appearance of a new superconducting order parameter at the lower transition. Similarly, global measurements of the magnetization [4] identify features keyed to the buried superconducting phase.

Samples prepared in a toroidal topology, monitored locally, can amplify any unusual vortex behavior. Flux will accumulate in the center and a local probe placed over the hole is sensitive to motion anywhere on the torus [5]. Moreover, circulation around a spatial singularity can change with changes in the superconducting order. This is most clearly seen in superfluid ${ }^{3} \mathrm{He}$, where phase transitions in the vortices [6] and the quenching of persistent currents around a physical obstruction [7] are both consequences of a degenerate order parameter with spin triplet pairing.

The situation in the solid state is not as clear-cut. Recent experiments have demonstrated that excess flux can be trapped for $T \leq T_{c 2}$ in a torus of polycrystalline $\mathrm{U}_{0.97} \mathrm{Th}_{0.03} \mathrm{Be}_{13}$ [8], the only other heavy fermion superconductor besides $\mathrm{UPt}_{3}$ with a double transition. The observed sensitivity of vortex pinning to magnetic field history may be a manifestation of broken time reversal invariance below $T_{c 2}$ [8], an interpretation bolstered by unambiguous evidence from muon spin relaxation studies [9] of local magnetic fields in the low-temperature superconducting state of $\mathrm{U}_{1-x} \mathrm{Th}_{x} \mathrm{Be}_{13}$ for $0.018<x<0.045$. In the case of $\mathrm{UPt}_{3}$, there are no reported measurements on crystals formed as tori, and the presence [10] or absence [11] of zero-field muon spin relaxation induced by superconductivity below $T_{c 2}$ remains a subject of debate.

In this paper, we take a direct approach to distinguishing the mechanisms of flux pinning in the two, zero-field superconducting states of $\mathrm{UPt}_{3}$ [12]. Using an array of miniature Hall probes, we measure the magnetization locally in crystals with toroidal topology, both for tori oriented parallel and perpendicular to the hexagonal basal plane. The magnetometer array permits a comparison of flux pinning in or near the hole, which may be sensitive to the proximity of an additional surface, with pinning in the bulk. In contrast to the global measurements of Amann et al. [4], our local probe reveals no feature in the remanent magnetization. However, we find an unusual instability in the pinning manifest only in the lower superconducting phase, with a sharp onset at $T_{c 2}$. Although the proximity to the hole enhances the instability, the absence of a hole does not destroy it. We model our results by considering the effects on the vortex creep of domains rising from broken time reversal symmetry and present computer simulations of the resulting irreversibility in the local magnetization.

Rectangularly shaped single crystals of typical dimensions $3 \times 2 \times 0.5 \mathrm{~mm}^{3}$ were grown by the vertical-floatzone refining method, annealed at $950{ }^{\circ} \mathrm{C}$ for $12 \mathrm{~h}$, and then slowly cooled [13]. We spark cut a hole $150 \mu \mathrm{m}$ in diameter clear through the sample along either the a or c axis. The sample was pressed in contact with a linear array of five InAs thin-film Hall probes [14] spaced $200 \mu \mathrm{m}$ apart, each with active area $100 \times 200 \mu \mathrm{m}^{2}$. By 
strategically positioning the array to span the sample from hole to edge, we could probe the local magnetization at any proximity to a surface, and with standard lock-in techniques, we were able to resolve fields to within $1 \mathrm{G}$. We cooled the crystals through $T_{c 1}$ to a fixed temperature in zero field, and cycled through a series of ten consecutive hysteresis loops at a $0.5 \mathrm{Oe} / \mathrm{s}$ ramp rate. A cycling field of 200 Oe was sufficient to minimize the turn-around portion of the hysteresis loop. After expelling the flux by warming above $T_{c 1}$, we repeated the procedure for a series of temperatures between 300 and $600 \mathrm{mK}$.

We plot in Fig. 1(a) magnetization hysteresis loops taken over the hole, in a crystal aligned with an aaxis hole, at $T_{c 2}<T=470 \mathrm{mK}<T_{c 1}$. These data are summarized in Fig. 1(b) as the mean and standard deviation, where for clarity, the standard deviation for the decreasing branch is plotted with its sign reversed. At this temperature, firmly inside the upper superconducting phase, the standard deviation loop is featureless. Contrast this with loops taken at $350 \mathrm{mK}$, shown in Fig. 2(a), inside the lower superconducting phase. Below $T_{c 2}$ the hysteresis loops exhibit an instability to avalanching in the region just beyond remanence, i.e., for $H$ between 0 and $75 \mathrm{Oe}$ on the increasing field branch and 0 to $-75 \mathrm{Oe}$ on the decreasing branch. This concentrated instability shows up clearly in the standard deviation shown in Fig. 2(b).

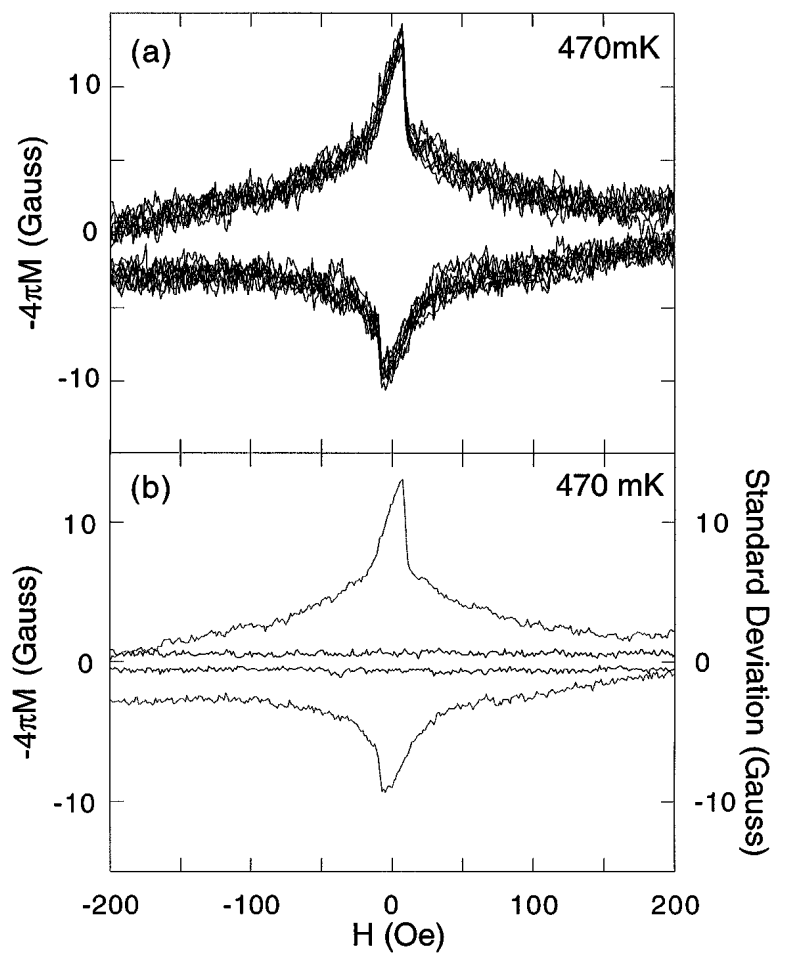

FIG. 1. (a) Ten magnetization hysteresis loops measured over the hole in a torus of $\mathrm{UPt}_{3}$ with an a-axis hole at $T_{c 2}<T=$ $470 \mathrm{mK}<T_{c 1}$. (b) The mean and the standard deviation of the hysteresis loops.
We plot in Fig. 3 the mean remanent magnetization itself on the same temperature scale as measurements of the specific heat, marking the positions of the two superconducting transitions (vertical lines). The remanent magnetization diminishes to zero at $T_{c 1}$, as expected. However, there is clearly no feature in the remanent magnetization coincident with $T_{c 2}$. The pinning increases with decreasing temperature, but it does not undergo any discontinuous change in strength in the lower superconducting phase. We observe the same behavior in the bulk region of this sample, in a separate crystal with a c-axis hole, and in a crystal with no hole.

The irreproducibility caused by the avalanche instability does appear to be sensitive to the low-temperature transition into a different superconducting state. In an attempt to quantify this effect, we introduce a metric which we term the "intrinsic noise." The total noise, measured as the standard deviation, is assumed to be an incoherent sum of the intrinsic noise and the systematic noise. Taking the root mean square difference between the standard deviation in the irreproducibility region and the standard deviation away from this region (a reasonable estimate of the systematic noise), we extract the intrinsic noise. Its temperature dependence, plotted in Fig. 4(a), is striking. The intrinsic noise is most pronounced at low temperatures, decreases linearly with increasing temperature, and drops effectively to zero right at $T_{c 2}$. As in the case of the remanent magnetization, the behavior of the intrinsic

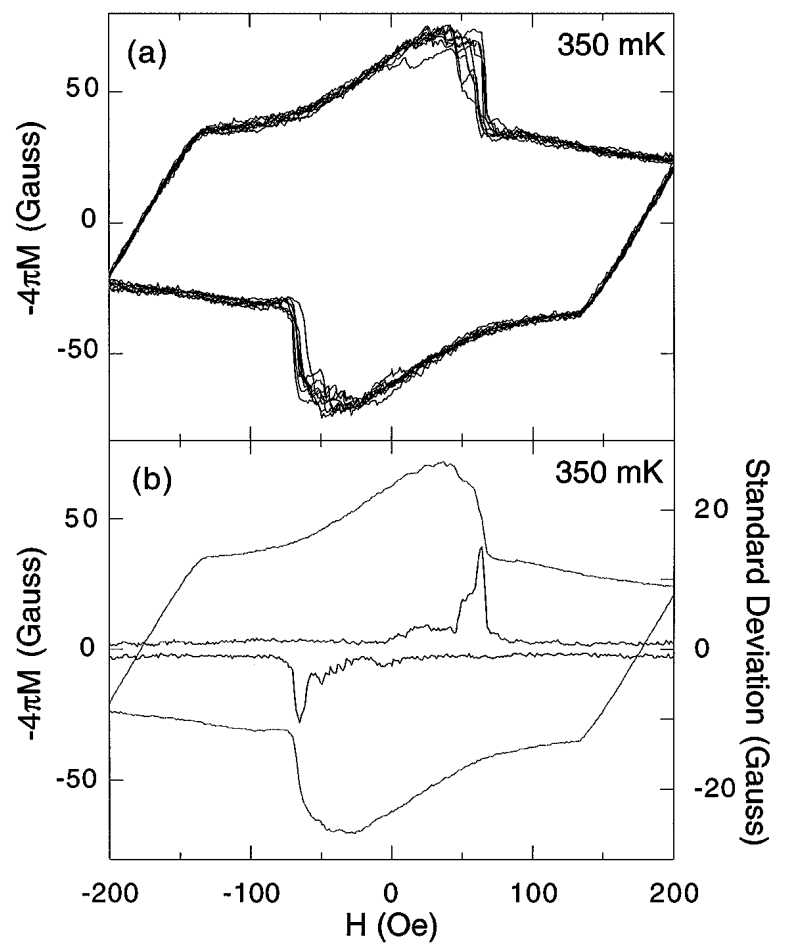

FIG. 2. Corresponding data to Fig. 1 at $T=350 \mathrm{mK}<T_{c 2}$. Here, irreproducible avalanches lead to a finite standard deviation for applied fields just beyond remanence. 


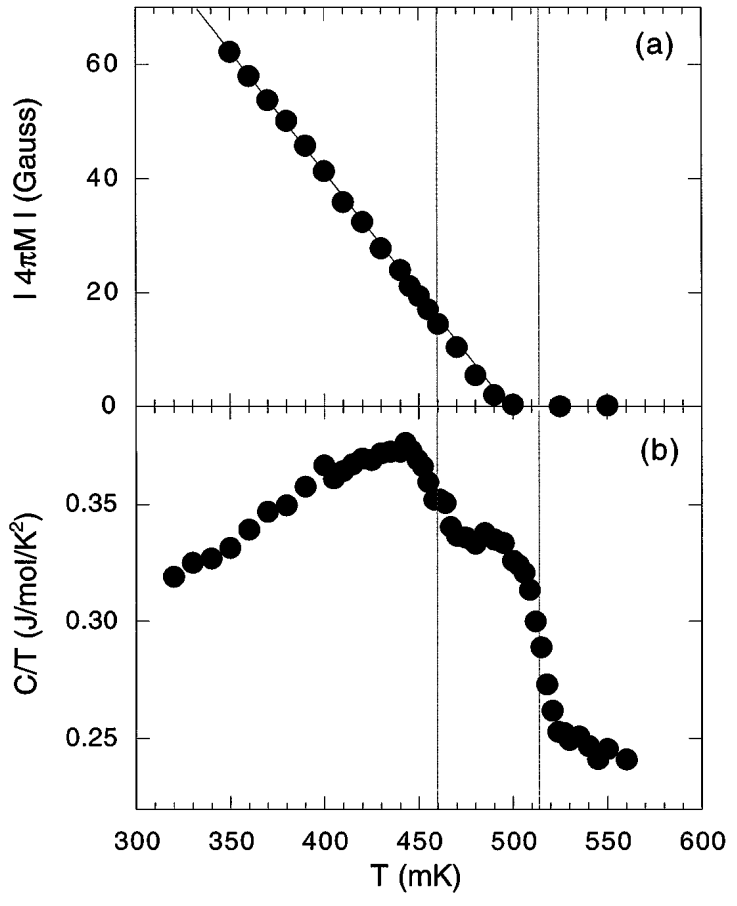

FIG. 3. (a) Remanent magnetization and (b) specific heat versus temperature. Vertical lines indicate the superconducting transitions from the jumps in the specific heat. $M(T)$ passes smoothly through $T_{c 2}$.

noise is robust. The development of a signal in the buried superconducting phase is seen in Fig. 4(b) for measurements over a hole along the $\mathbf{c}$ axis, and it persists in the bulk region of the a-axis hole crystal [Fig. 4(c)], although with significantly reduced magnitude. In all the samples, the onset of the instability coincides with the jump in the specific heat at $T_{c 2}$.

Vortex avalanches and irreversible flux flow are standard features in type-II superconductors. The mystery here is what changes at the lower superconducting transition. Even though there can be additional barriers to flux entry at the surface of the hole, $M(T)$ varies smoothly for all $T<T_{c 1}$ (Fig. 3) and there is only a subtle modulation of $d H_{c 1} / d T$ through $T_{c 2}$ [2,3]. One possibility is that broken time reversal symmetry, a general feature of most proposed theories for superconducting $\mathrm{UPt}_{3}$ [1], may have observable consequences on flux motion and pinning. The order parameter in the high-temperature superconducting phase is usually assumed to be nondegenerate, while the low-temperature phase breaks time reversal symmetry and is at least twofold degenerate. Domains of different superconducting states can be formed below $T_{c 2}$, separated by domain walls which are pinned at impurities. These domain walls can pin vortices and, consequently, can create barriers to flux motion. In particular, vortices on domain walls between time reversal symmetry breaking states can decay into two fractional vortices $[15,16]$. These fractional vortices can reside only on the domain

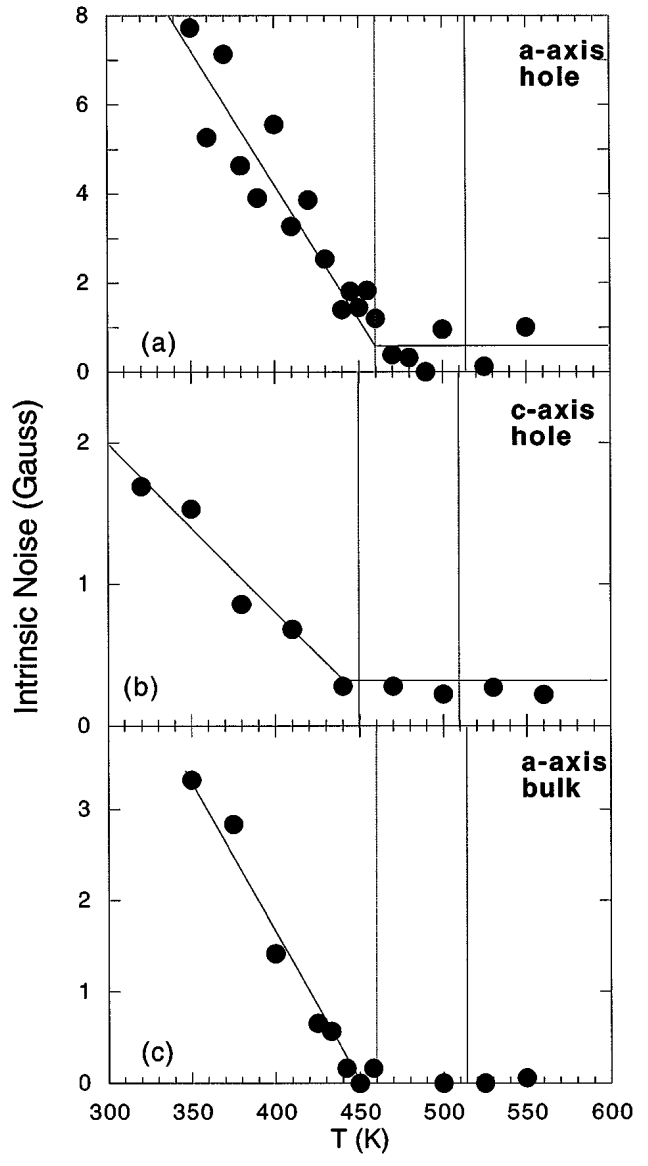

FIG. 4. Intrinsic noise versus temperature for local measurements along (a) the a axis over the hole, (b) the c axis over the hole, and (c) the a axis away from the hole. In all cases, the onset of the instability coincides with $T_{c 2}$.

walls and are capable of drastically enhancing the barrier effect because of their repulsive interaction with other vortices.

We have investigated the effect of such barriers on the magnetization process of a superconductor in a computer simulation. In a simple model, we consider a system with domain walls located symmetrically around the hole of the torus so that they act like a fence around the center. Using the Bean model of the critical state in a dirty superconductor [17], we include both a finite $H_{c 1}$ as the threshold for the entrance of flux lines from the exterior of the system and a barrier field $H_{b}$, which must be exceeded by the local field (proportional to vortex concentration) for vortices to pass through the domain wall. When vortices first enter the sample, the center region remains empty until $H_{b}$ is reached at the domain walls. At this point, flux can enter the center region, following the Bean profile everywhere. With the reversed field, the vortices in the center region are trapped. Once the negative critical field $H_{c 1}$ is reached, vortices in the outer part are replaced by inverted vortices. However, the vortices in the center only can be annihilated and replaced by inverted vortices when 


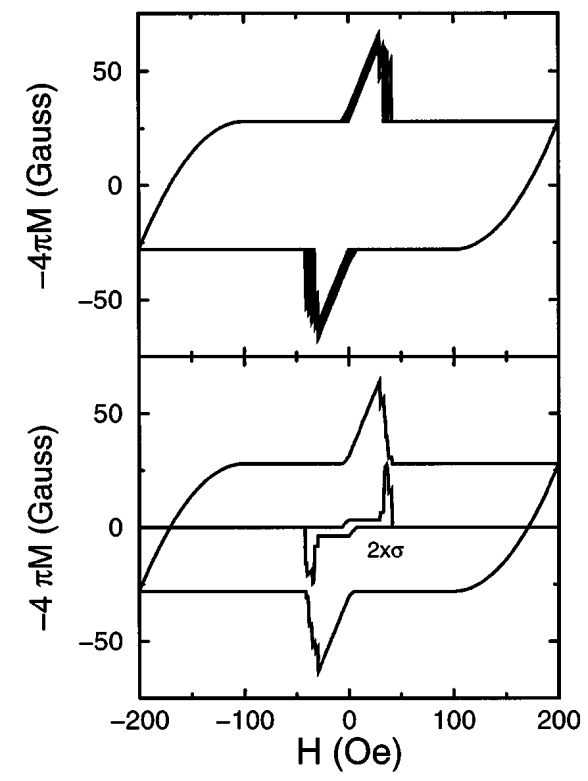

FIG. 5. Computer simulations of magnetization hysteresis loops, assuming pinning from domain walls due to broken time reversal symmetry below $T_{c 2}$. See text for details.

the external field reaches a sufficiently large negative value that $H_{b}$ is exceeded at the domain wall. Inverted vortices then can enter freely, leading to a fast flip of the magnetization in the center and avalanches.

The noise and irreversibility can originate from the variation of the position of the domain walls. During the field cycle, the domain walls may move slightly under the pressure of the vortices. Avalanches in the center region should occur at different external fields for different domain wall positions, leading to deviations between consecutive cycles. We plot in Fig. 5 the results of a computer simulation where we have placed the domain walls randomly for every field cycle. The cycles show noise at the same positions as in the experiment, i.e., close to (mainly above) $H_{c 1}$. Additionally, we find in our simulation that the noise shrinks when we lower $H_{b}$. The barrier should become weaker when the temperature approaches $T_{c 2}$ from below, because the domain wall width grows such that pinning effects are weakened and fractional vortices become less stable. This aspect agrees qualitatively with the experiment. At present, however, it is difficult to argue from our model for a linear dependence of the intrinsic noise on $\left(T_{c 2}-T\right)$, since the temperature dependence of the barrier field can depend on numerous factors.

In summary, local measurements of the magnetization in a torus of $\mathrm{UPt}_{3}$ reveal a sharp change in the stability of flux pinning at the lower superconducting transition temperature. The effect is amplified by the toroidal topology, and it can be observed over the bulk as well as over the hole, both parallel and perpendicular to the hexagonal basal plane. Broken time reversal symmetry below $T_{c 2}$ provides a natural mechanism for the observed avalanching, as supported by simulation. Finally, we observe no discontinuity at the lower transition in the strength of the pinning, characterized by the remanent magnetization.

We are grateful to D. G. Hinks for providing the crystals of $\mathrm{UPt}_{3}$. The work at the University of Chicago was supported by NSF No. DMR95-07873. E.S. acknowledges support from the National Science Foundation (DMR91-20000) through the Science and Technology Center for Superconductivity.

[1] For recent reviews, see J. A. Sauls, Adv. Phys. 43, 113 (1994); R. H. Heffner and M. R. Norman, Comments Condens. Matter Phys. 17, 361 (1996).

[2] B. S. Shivaram, J. J. Gannon, Jr., and D. G. Hinks, Phys. Rev. Lett. 63, 1723 (1989).

[3] E. Vincent, J. Hammann, L. Taillefer, K. Behnia, N. Keller, and J. Flouquet, J. Phys. Condens. Matter 3, 3517 (1991).

[4] A. Amann, P. Visani, K. Aupke, A. C. Mota, M. B. Maple, Y. Dalichaouch, P. E. Armstrong, and Z. Fisk, Europhys. Lett. 33, 303 (1996).

[5] E. R. Nowak, O.W. Taylor, L. Liu, H. M. Jaeger, and T. Selinder, Phys. Rev. B 55, 11702 (1997); S. Field, J. Witt, and F. Nori, Phys. Rev. Lett. 74, 1206 (1995).

[6] For a review, see P. Hakonen, O. V. Lounasmaa, and J. Simola, Physica (Amsterdam) 160B, 1 (1989).

[7] P. L. Gammel, H.E. Hall, and J. D. Reppy, Phys. Rev. Lett. 52, 121 (1984); J.P. Pekola, J. T. Simola, K. K. Nummila, O. V. Lounasmaa, and R. E. Packard, ibid. 53, 70 (1984); J. C. Davis, J. D. Close, R. Zieve, and R.E. Packard, ibid. 66, 329 (1991).

[8] R. J. Zieve, T. F. Rosenbaum, J. S. Kim, G. R. Stewart, and M. Sigrist, Phys. Rev. B 51, 12041 (1995).

[9] R. H. Heffner, J.L. Smith, J. O. Willis, P. Birrer, C. Baines, F. N. Gygax, B. Hitti, E. Lippelt, H. R. Ott, A. Schenck, E. A. Knetsch, J. A. Mydosh, and D.E. MacLaughlin, Phys. Rev. Lett. 65, 2816 (1990).

[10] G. M. Luke, A. Keren, L.P. Le, W.D. Wu, Y. J. Uemura, D. A. Bonn, L. Taillefer, and J. D. Garrett, Phys. Rev. Lett. 71, 1466 (1993).

[11] P. Dalmas de Reotier, A. Huxley, A. Yaouanc, J. Flouquet, P. Bonville, P. Imbert, P. Pari, P. C. M. Gubbens, and A. M. Mulders, Phys. Lett. A 205, 239 (1995).

[12] For a study of pinning in the high field $C$ phase, see $B$. Ellman and L. Taillefer, Phys. Rev. B 56, R5767 (1997).

[13] D. S. Jin, S. A. Carter, B. Ellman, T. F. Rosenbaum, and D. G. Hinks, Phys. Rev. Lett. 68, 1597 (1992).

[14] E. Pugel, E. Shung, T. F. Rosenbaum, and S. P. Watkins, Appl. Phys. Lett. 71, 2205 (1997).

[15] M. Sigrist, T. M. Rice, and K. Ueda, Phys. Rev. Lett. 63, 1727 (1989); M. Sigrist and K. Ueda, Rev. Mod. Phys. 63, 239 (1991).

[16] G. Volovik and L. P. Gor'kov, Pis'ma Zh. Eksp. Teor. Fiz. 39, 550 (1984) [JETP Lett. 39, 674 (1984)].

[17] C. P. Bean, Phys. Rev. Lett. 8, 250 (1962). 